\title{
Stabilization of Switched Systems Using Only A Single Fractional Order PI Controller
}

\author{
S. E. Hamamci and I. Işık
}

\begin{abstract}
In this study, stabilization of switched systems is investigated using only a single controller. Due to providing a rich variety in the control performance, fractional order PI controller is selected as stabilizing controller. For the stabilization process, well known D-partition method is utilized. The proposed method is based on obtaining stability regions in $\left(k_{p}, k_{i}\right)$-plane for each sub-system of switched system and then determining a common stability region from intersection of these stability regions. The common region includes the controllers making the overall closed loop system stable for all sub-systems. By changing the order of the integrator in the controller fractionally, a set of common stability regions are obtained. A switched system with two sub-systems was simulated to demonstrate the efficiency of the method.
\end{abstract}

Index Terms - Switched systems, Fractional order PI controller, Stabilization, Common stability region.

\section{INTRODUCTION}

$\mathrm{S}$ WITCHED systems are a category of hybrid systems composed of different sub-systems and a switching rule specifying the active sub-system in the timetable. [1-3]. The most important property of these systems is to have a decisive impact of switching operation on behavior of the whole system. Although all of the sub-systems in a switched system are stable, the switching operation may lead to unstable or chaotic behaviors in the overall system. Similarly, a switched system with some unstable sub-systems may exhibit a stable behavior with the switching operation [4]. There are many application areas of the switched systems in control of industrial systems and modeling of engineering and physical systems like the switching power converters, automotive industry, aircraft and air traffic control, communication systems, and many other fields [5-7 and references therein]. The most important reason of this, a lot of system can be expressed as "having the switching dynamic between the local models" [4].

Recently, many studies have been reported about the switched systems in literature. In general, these studies can be grouped under two main headings. One of them is analysis of

S. E. HAMAMCI is with the Department of Electrical-Electronics Engineering Department, Inonu University, Malatya, Turkey (e-mail: serdar.hamamci@inonu.edu.tr).

I. IŞIK is with the Department of Electrical-Electronics Engineering Department, Inonu University, Malatya, Turkey (e-mail: ibrahim.isik@inonu.edu.tr). switching system dynamics that is mostly focused on stability $[1,5,8]$ and controllability/observability $[9,10]$ issues. On the other hand, second one is control system design which supply desired performance. There are some studies on the control of switched systems in literature [11-14]. In these studies, the numbers of the used controllers are as the number of subsystems generally. However, HosseinNia et al [15] employed only a single controller for the switched systems. The utilization of single controller is an important idea because of providing the easier design effort.

In this study, the switched systems and their control with single fractional order PI controller is investigated. The objective of the control is to obtain all stabilizing set of fractional order PI controllers for the overall switched system rather than better performance. First, the stability regions using the fractional order PI controller for each sub-systems are obtained separately by Neimark's D-partition method [16]. This region includes the fractional order PI controller parameters that stabilize their sub-systems. Then, plotting these stability regions on a same graph, the common stability region of fractional order PI controller which makes the overall switched system stable are obtained by intersecting these stability regions. Every pair of $\left(k_{p}, k_{i}\right)$ in this common region gives the stable closed loop control response for each instant of switching. Finally, a set of common stability regions for the various values of fractional order in the controller is determined. An important advantage of the method is that the designer eliminates the controllers which make the overall switched control system unstable.

\section{SWITCHED CONTROL SYSTEM}

In literature, the most commonly used control system structure for the switched systems shown in Fig. 1 was suggested by Liberzon and Morse [17]. This structure contains the same number of sub-systems and sub-controllers. In each switching period, only one sub-system and one sub-controller are active. Switching unit decides which the pair of subsystem and sub-controller will be selected. But the major disadvantage of this structure for the designer is to design the sub-controllers as many as the number of sub-systems. Therefore, HosseinNia et al [15] suggested an alternative control system structure shown in Fig. 2 instead of the structure of Liberzon and Morse. As can be seen from the figure, the design of only one controller for the switched system control will be sufficient. However, the major 
disadvantage of this structure for the designer is to design a controller which must control all the sub-system is very difficult. But HosseinNia et al showed in their publications [15] and [18] that the successful control results can be obtained if the suitable design methods are developed.

In a switched linear system, the transfer function corresponding to each sub-system is defined as

$G_{i}(s)=\frac{N_{i}(s)}{D_{i}(s)}=\frac{\sum_{k=0}^{p} b_{i k} s^{k}}{\sum_{k=0}^{q} a_{i k} s^{k}} \quad i=1 \sim n$

where $i$ determines which sub-system is active, $k$ refers to indices of polynomial coefficients and $n$ denotes the number of sub-systems. Here $N_{i}(s)$ and $D_{i}(s)$ are the numerator and denominator polynomials of $G_{i}(s) . p$ and $q$ are the degrees of the polynomials $N_{i}(s)$ and $D_{i}(s)$, respectively. In (1), the condition $p<q$ must be fulfilled for the strictly properness property. The characteristic polynomial of the control system changes for each position of switching. Therefore, the characteristic polynomial for any switching status is described as

$P_{i}(s)=1+G_{i}(s) C(s) \quad i=1 \sim n$.

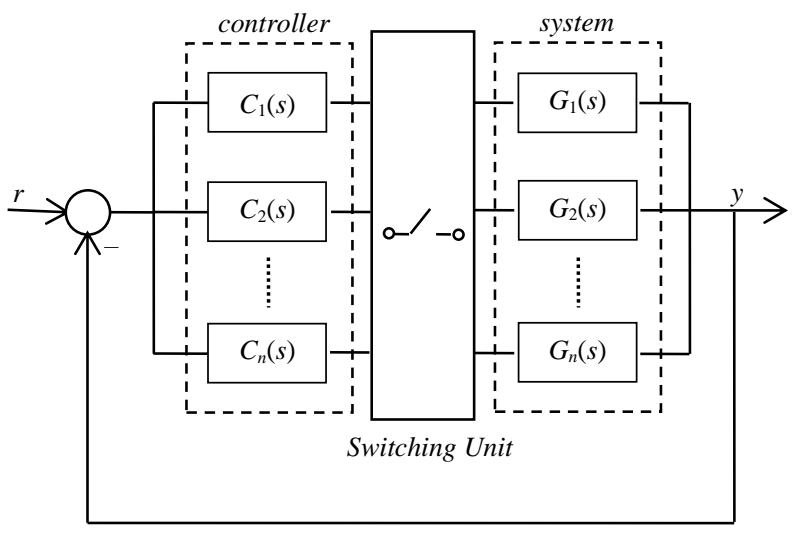

Fig. 1. Switched control system structure containing multi controller.

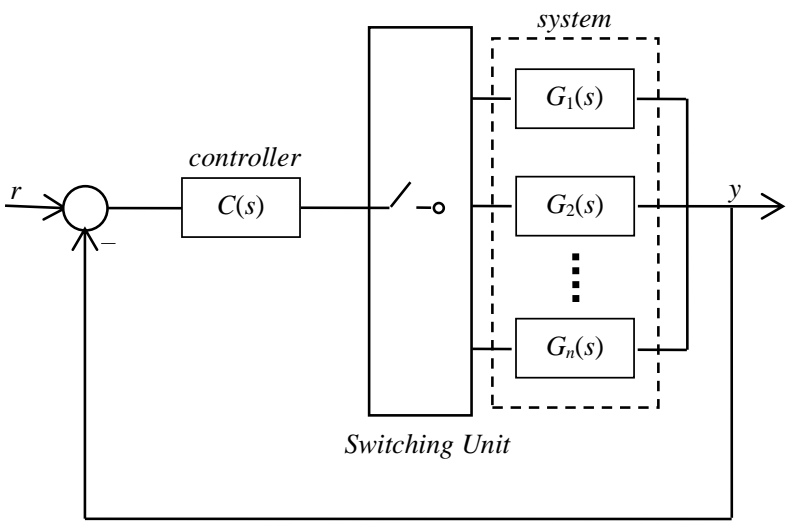

Fig. 2. Switched control system structure containing only one single controller.
Each characteristic polynomial must be Hurwitz stable for the stability of overall closed-loop system.

The controller to be used in this study is in the type of fractional order PI controller [19] and defined by

$C(s)=k_{p}+\frac{k_{i}}{s^{\lambda}}=\frac{k_{p} s^{\lambda}+k_{i}}{s^{\lambda}}$.

where $\lambda$ is fractional order of the integrator part of the controller and its value may vary in the range of $(0,2)$. This is an advantage because the time and frequency responses can be shaped using functions and, as a consequence, the performance of the closed loop can be improved over the use of integer-order controllers [20]. For $\lambda=1$, the fractional order PI controller turns into the classical integer order PI controller.

\section{COMPUTATION OF ALL COMMON STABILITY REGIONS}

It is well known that the stability of a control system is determined by locating it's all poles to the left half plane (LHP) of the complex plane. The stability region for the control system is expressed by a conformal mapping of the LHP to the controller parameter plane. This region contains a controller parameters set that makes the closed loop system stable. Therefore, the characteristic polynomial of the control system plays an important role for obtaining the stability region.

As mentioned above, the switched control system has many characteristic polynomials as defined in (2). Substituting the transfer function of the switched system in (1) and the controller transfer function in (3) into (2), we obtain

$$
\begin{aligned}
P_{i}(s) & =1+\frac{\sum_{k=0}^{p} b_{i k} s^{k}}{\sum_{k=0}^{q} a_{i k} s^{k}} \cdot \frac{k_{p} s^{\lambda}+k_{i}}{s^{\lambda}} \\
& =\sum_{k=0}^{q} a_{i k} s^{k+\lambda}+k_{p} \sum_{k=0}^{p} b_{i k} s^{k+\lambda}+k_{i} \sum_{k=0}^{p} b_{i k} s^{k} .
\end{aligned}
$$

For obtaining the stability regions, there are some methods in the literature, for example parameter space approach [21], Hermite Biehler theorem [22], D-partition method [16], etc. In this study, the D-partition method is preferred because of its easy and systematic methodology. The method is based on the calculation of stability boundaries as summarized in Appendix after Section 5. Hence, for each sub-systems, the real root boundary (RRB) line putting $s=0$ into (4) is obtained as

$k_{i}=0$.

The infinite root boundary does not exist because of $m<n$. Finally, the complex root boundary (CRB) is obtained by equating the real and imaginary parts of $P_{i}(\mathrm{j} w)$ to zero. In this case, Equation (4) is returned to two equations:

$w^{\lambda} A(w) k_{p}+B(w) k_{i}=w^{\lambda} P(w)$

$w^{\lambda} C(w) k_{p}+D(w) k_{i}=w^{\lambda} Q(w)$

where, 
$A(w)=\sum_{k=0}^{p} b_{i k} x_{k} w^{k}$

$B(w)=\sum_{k=0}^{p} b_{i k} z_{k} w^{k}$

$C(w)=\sum_{k=0}^{p} b_{i k} y_{k} w^{k}$,

$D(w)=\sum_{k=0}^{p} b_{i k} t_{k} w^{k}$,

$P(w)=-\sum_{k=0}^{q} a_{i k} x_{k} w^{k}$,

$Q(w)=-\sum_{k=0}^{q} a_{i k} y_{k} w^{k}$,

$x_{k}=\Re\left\{j^{(k+\lambda)}\right\}, y_{k}=\Im\left\{j^{(k+\lambda)}\right\}, z_{k}=\Re\left\{j^{k}\right\}$ and $t_{k}=\Im\left\{j^{k}\right\}$. Here,

$\mathfrak{R}$ and $\mathfrak{J}$ denote the real and imaginary parts of a complex number or variable. After solving these two equations, we obtain $k_{p}$ and $k_{i}$ parameters with the following equations:

$k_{p}=[Q(w) B(w)-P(w) D(w)] /[B(w) C(w)-A(w) D(w)]$,

$k_{i}=w^{\lambda}\{[Q(w) B(w)-P(w) D(w)] /[B(w) C(w)-A(w) D(w)]\}$.

Changing $w$ between $(0, \infty)$, many $\left(k_{p}, k_{i}\right)$ points can be marked as a point in $\left(k_{p}, k_{i}\right)$-plane. Connecting these points, the complex root boundary can be obtained as a line or curve.

Plotting complex and real root boundaries together on a same $\left(k_{p}, k_{i}\right)$-plane, the parameter plane is divided into a large number of parts. The most important feature of these parts is that all $\left(k_{p}, k_{i}\right)$ pairs in a region are produce the characteristic polynomials at the same stability property. Therefore, these parts can be tested by substituting any test point, which is selected randomly from each part, into characteristic polynomial. The part is determined as the stability region whose all roots of the characteristic polynomial are in LHP.

For $i=1 \sim n$, a set of stability regions corresponding to each subsystems is found. Intersection of these stability regions are called as common stability region. Any $\left(k_{p}, k_{i}\right)$ pairs chosen in the common region make the control system stable for all situations of switch. Similarly, changing the order of integrator in controller in the range of $(0,2)$, a set of common stability regions is determined. As a result, the designer has a large facility for the performance decision.

\section{SIMULATION EXAMPLE}

In this section, a simulation example to show the efficiency of the proposed method will be given. Since the widely used switched systems in industry are with two sub-systems, a switched system having two sub-system with second order considered in [15] is studied in this example. The transfer functions of the system is given by

$$
\begin{aligned}
& G_{1}(s)=\frac{2}{s^{2}+0.6 s+0.1} \\
& G_{2}(s)=\frac{0.5}{s^{2}+0.3 s+0.023}
\end{aligned}
$$

The purpose of this example is to obtain the common stability regions for different values of $\lambda$.

The characteristic polynomials for the sub-systems can be obtained as

$$
\begin{aligned}
& P_{1}(s)=s^{\lambda+2}+0.6 s^{\lambda+1}+\left(0.1+2 k_{p}\right) s^{\lambda}+2 k_{i} \\
& P_{2}(s)=s^{\lambda+2}+0.3 s^{\lambda+1}+\left(0.023+0.5 k_{p}\right) s^{\lambda}+0.5 k_{i}
\end{aligned}
$$

The RRB for both $G_{1}(s)$ and $G_{2}(s)$ are the line $k_{i}=0$ as given in (5). From (8) and (9), the CRB for each sub-systems are calculated as below:

- Sub-system 1:

$k_{p}=\frac{1}{2 y_{0}}\left[-y_{2} w^{2}-0.6 y_{1} w-0.1 y_{0}\right]$

$k_{i}=\frac{w^{\lambda}}{2 y_{0}}\left[\left(x_{0} y_{2}-x_{2} y_{0}\right) w^{2}-0.6\left(x_{0} y_{1}-x_{1} y_{0}\right) w\right]$

- Sub-system 2:

$k_{p}=\frac{2}{y_{0}}\left[-y_{2} w^{2}-0.3 y_{1} w-0.023 y_{0}\right]$

$k_{i}=\frac{2 w^{\lambda}}{y_{0}}\left[\left(x_{0} y_{2}-x_{2} y_{0}\right) w^{2}-0.3\left(x_{0} y_{1}-x_{1} y_{0}\right) w\right]$

where $x_{2}+\mathrm{j} y_{2}=\mathrm{j}^{(\lambda+2)}, x_{1}+\mathrm{j} y_{1}=\mathrm{j}^{(\lambda+1)}$ and $x_{0}+\mathrm{j} y_{0}=\mathrm{j}^{\lambda}$.

In case of $\lambda=1$, the view of RRB and CRB in the parameter plane are given in Figs. 3 and 4 for each sub-systems. It is obviously seen that the parameter planes in these figures is partitioned to three parts:

1. above of CRB,

2. between CRB and RRB,

3. under of RRB.

When the stability of these parts are examined for each $\left(k_{p}, k_{i}\right)$ points taken from the parts randomly, one concludes that the triangular region is stable between CRB and RRB for both of sub-systems. Note that there is not any boundary at the right side of this triangular region. It is seen from the figures, the stability region of first sub-system is greater than that of second sub-system. This implies that the number of PI controllers making the first sub-system stable is greater than ones for making the second sub-system stable. If Figs. 3 and 4 are plotted on a same frame as shown in Fig. 5, the intersection of two stability regions $\left(1^{\text {st }}\right.$ region in Fig. 5) is called as common stability region. The PI controllers selected from this region makes the overall closed-loop system stable in every switching stage. Thus the designer can choose any PI controller from the common region without considering the switching status and the subsystems. In the second region drawn with horizontal lines and outside of common region in

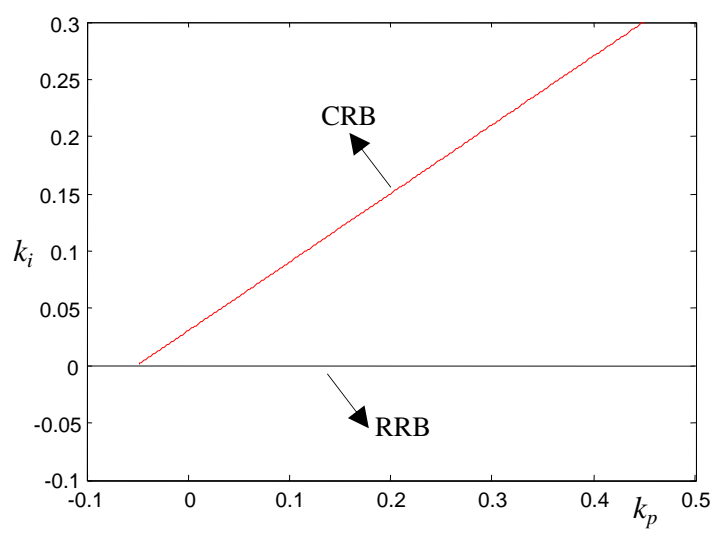

Fig. 3. PI stability region belonging to $G_{1}(s)$ sub-system. 


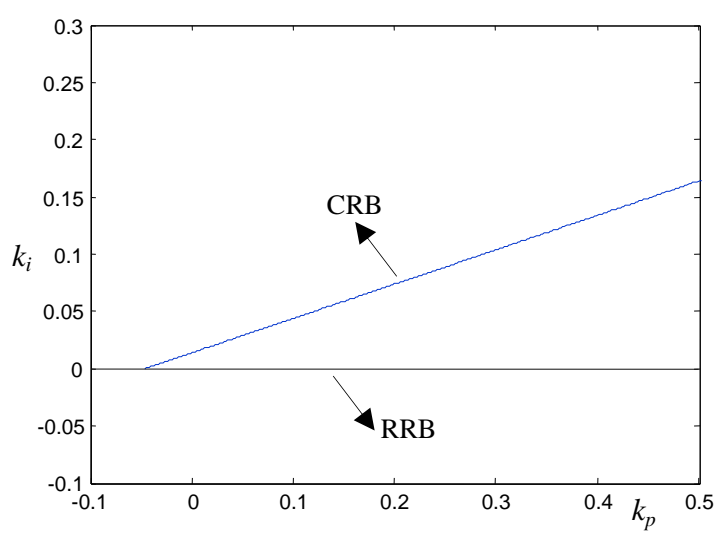

Fig. 4. PI stability region belonging to $\mathrm{G}_{2}(\mathrm{~s})$ sub-system.

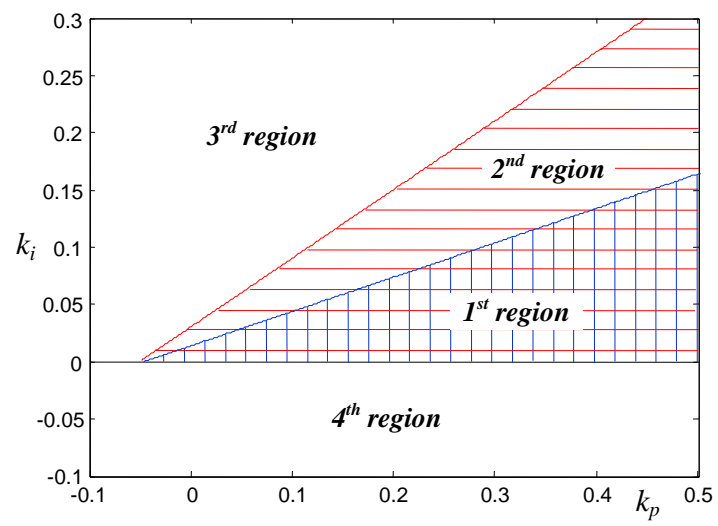

Fig. 5. Common stability region of the overall control system.

Fig. 5, the overall control system is stable for first sub-system but unstable for the second sub-system. In the third and fourth regions where they are not drawn with any lines, the control system is unstable for both of sub-systems. Furthermore, it is seen from Fig. 5 that there is not any region in the parameter space where the overall control system is stable for the second sub-system and is unstable for the first sub-system.

To verify the results in Fig.5, the unit step responses for the PI controllers $(\lambda=1)$ selected from four different places of Fig. 5 are analyzed. In this analysis, the unit step signal is applied to the input of control system and switching from first subsystem to second sub-system is made at $t=75 \mathrm{~s}$. Firstly, the unit step response of the overall control system for the PI controller parameters which are selected as $k_{p}=0.05, k_{i}=0.01$ from the common stability region $\left(1^{\text {st }}\right.$ region) randomly is shown in Fig. 6a It is clearly seen from the figure, stable responses for the overall control system are produced by both first sub-system/PI controller pairs at first $75 \mathrm{~s}$ and second subsystem/PI controller pairs at next $75 \mathrm{~s}$. In Fig. $6 \mathrm{~b}$, the unit step response of the overall control system for $k_{p}=0.3, k_{i}=0.1$ values selected on boundary line between first and second regions is given. In this case, the control system is stable for the first $75 \mathrm{~s}$ but an oscillating response is occurred after switching. On the other hand, in Figs. $6 \mathrm{c}$ and $6 \mathrm{~d}$, the output

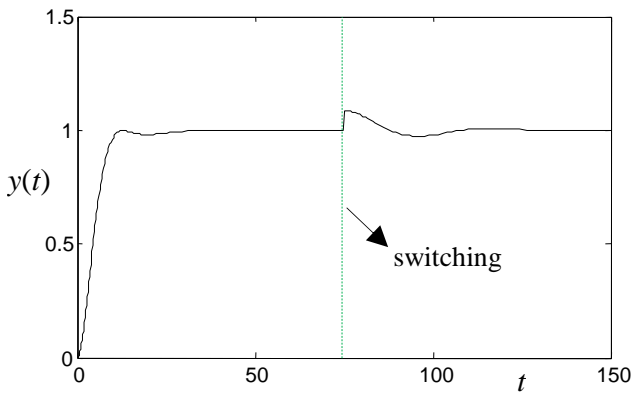

(a)

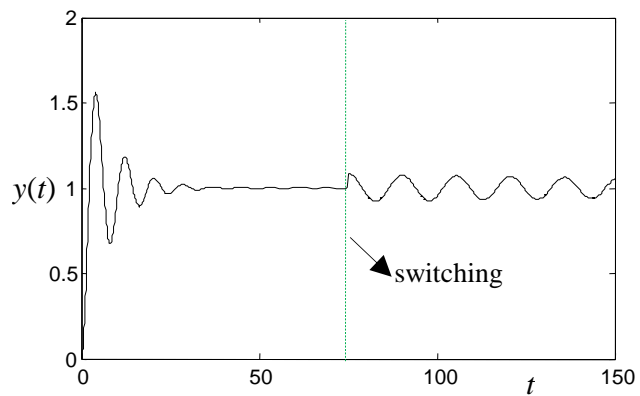

(b)

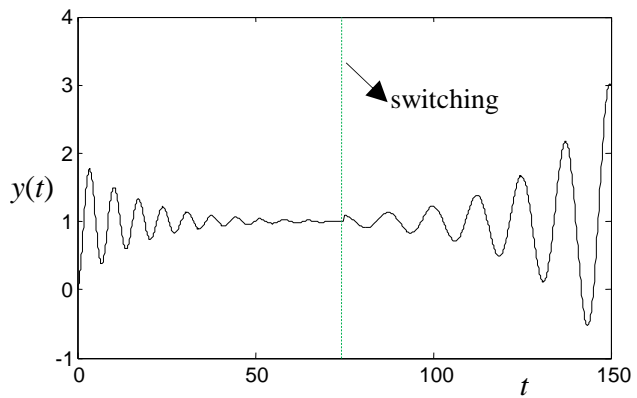

(c)

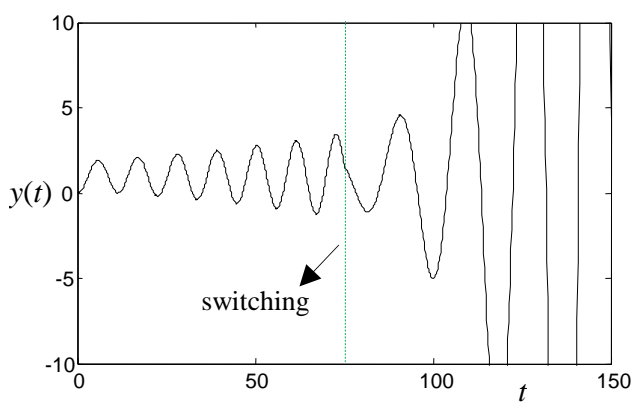

(d)

Fig. 6. Unit step responses of switched control system for four different PI controllers: a) $\mathrm{k}_{\mathrm{p}}=0.05, \mathrm{k}_{\mathrm{i}}=0.01$; b) $\mathrm{k}_{\mathrm{p}}=0.3, \mathrm{k}_{\mathrm{i}}=0.1$; c) $\mathrm{k}_{\mathrm{p}}=0.4, \mathrm{k}_{\mathrm{i}}=0.2$ and d) $\mathrm{k}_{\mathrm{p}}=0.1, \mathrm{k}_{\mathrm{i}}=0.1$.

responses of closed loop system are given for $k_{p}=0.4, k_{i}=0.2$ and $k_{p}=0.1, k_{i}=0.1$ values selected from second and third regions. Unit step response of the overall control system is stable before switching and unstable after the switching for PI controller values selected from second region while the unit step response of the control system is unstable before and after switching for the PI controller values chosen from third region. 


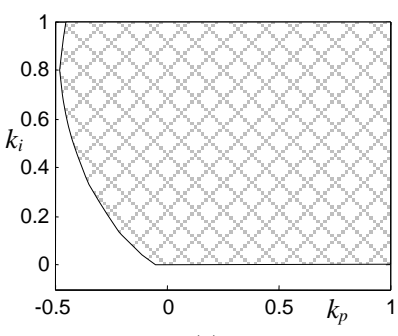

(a)

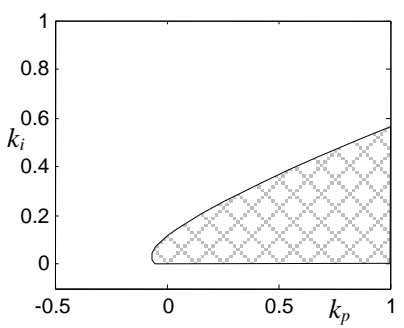

(c)

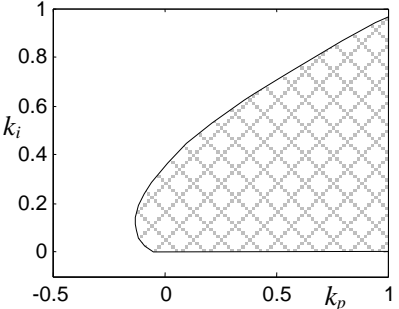

(b)

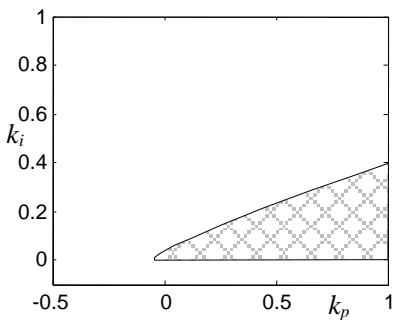

(d)

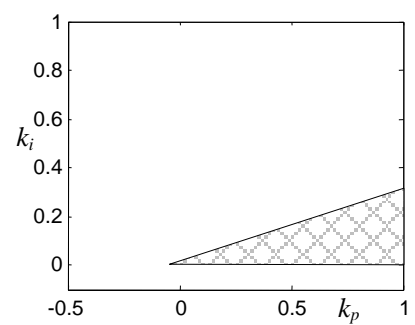

(e)

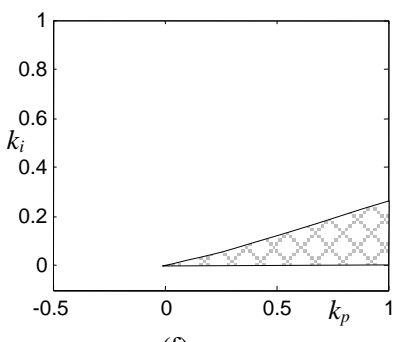

(f)

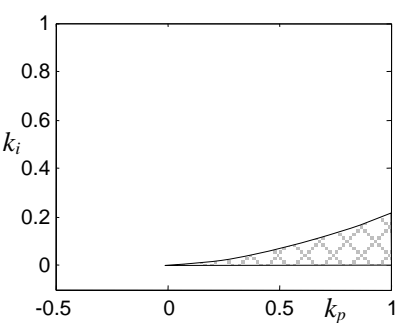

(h)

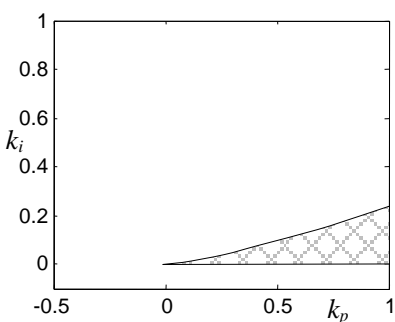

(g)

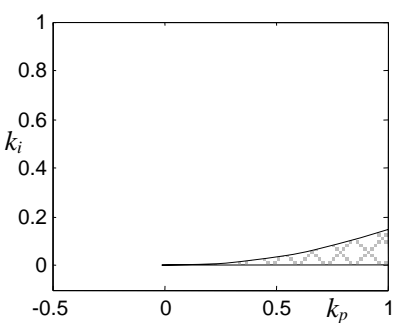

(i)
Fig. 7. The common stability regions for the values of $\lambda$ in the range of $(0,2)$ : a) $\lambda=0.2$, b) $\lambda=0.4$, c) $\lambda=0.6$, d) $\lambda=0.8, \quad$ e) $\lambda=1$, f) $\lambda=1.2$, g) $\lambda=1.4$, h) $\lambda=1.6$, i) $\lambda=1.8$.

Finally, changing $\lambda$ from 0 to 2 , the set of common stability regions are obtained as shown in Fig. 7. It is seen from Fig. 7 that smaller $\lambda$ gives bigger common stability region.

Remark: The fractional order PI controllers with smaller value of $\lambda$ than 1 provide bigger stability regions according to integer order PI controller. This is an important result that can give new ideas to the designers in industry.
The obtained results clearly show that the presented method gives a successful way to find a stability map for the fractional order PI switched control system.

\section{CONCLUSIONS}

In this paper, a study on obtaining the stability map for the switched systems using a single fractional order PI controller is given. The common stability region presented in this study contains all controllers which make the overall control system stable without considering the switching status. For obtaining the common stability region, it is made use of D-partition method. The method in this paper may be considered as an extended version of the method given in [23]. In the previous version, the switched systems with only two subsystems and the conventional PI controller are considered. In this paper, however, the switched systems with more subsystems and the fractional order PI controller which is generalized version of conventional PI controller are examined. Thus, a set of common stability regions using fractional PI controller is obtained instead of only a common stability region using the classical PI controller. This provides to the designer more flexibility for his/her decision in the controller selection process. Simulation results confirm the results. However, note that the performance of the overall switched control system is a different topic. Whether bigger stability region gives better performance or not is an open issue that must be studied.

\section{APPENDIX: SUMMARY FOR D-PARTITION METHOD}

Consider a characteristic polynomial with unknown $x_{i}$ $(i=1 \sim \mathrm{n})$ parameters which is defined by

$P\left(s ; x_{1}, x_{2}, \ldots x_{n}\right)=P_{0}(s)+P_{1}(s) x_{1}+\cdots+P_{n}(s) x_{n}$.

Here, $P_{i}(s)$ refers to known parts of polynomial for $i=1 \sim n$. To find $x_{i}$ unknown values, the D-partition method has been proposed by Neimark [16] as an effective technique which finds a set of $x_{i}$ values stabilizing the polynomial in (A.1). In other words, all the set of $x_{i}$ values are found so that all roots of the polynomial are remained in LHP. This method gives very good results in stability analysis and design of control systems. These sets of $x_{i}$ values constitute a stability region in $\left(x_{1}, x_{2}, \ldots, x_{n}\right)$-parameter space.

For obtaining the stability region in the parameter space, three important stability boundaries are used [21, 24]. These regions are defined as follows.

- Real Root Boundary (RRB): This boundary is determined by the equation $\left.P\left(s ; x_{1}, x_{2}, \ldots \ldots x_{n}\right)\right|_{s=0}=0$. This equation always gives a line in the parameter space if this boundary exists.

- Infinite Root Boundary (IRB): This boundary is obtained by the equation $\left.P\left(s ; x_{1}, x_{2}, \ldots \ldots x_{n}\right)\right|_{s=\infty}=0$. The equation means equating the largest coefficient of the polynomial to zero. This equation also always gives a line in the parameter space if this boundary exists.

- Complex Root Boundary (CRB): This boundary is found by the equation $\left.P\left(s ; x_{1}, x_{2}, \ldots \ldots x_{n}\right)\right|_{s=j w}=0$. For obtaining this boundary, putting $\mathrm{j} w$ instead of $s$ in the characteristic polynomial and equating the real and imaginary parts of the 
polynomial to zero separately. This equation usually gives a curve (sometimes a line in the simple systems) in the parameter space if this boundary exists.

These boundaries are partitioned the parameter space to many parts. Stability test is applied for each parts. In the results of the stability test, the stability region can be obtained.

\section{REFERENCES}

[1] D. Liberzon, Switching in Systems and Control, Birkäuser, 2003.

[2] J. Daafouz, P. Riedinger, and C. Lung, "Stability analysis and control synthesis for switched systems: A switched Lyapunov function approach," IEEE Trans. on Automatic Control, vol. 47, no. 11, pp. 1883-1887, 2002.

[3] S.L. Chen, Y.Yao, and X. Di, "Robust Stabilization for a Class of Uncertain Discrete-time Switched Linear Systems," In: Discrete Time Systems, edited by M.A. Jordán, InTech-Open Access Company.

[4] D.J. Leith, R.N. Shorten, W.E. Leithead, O. Mason, and P. Curran, "Issues in the design of switched linear control systems: A benchmark study," Int. J. Adapt. Control Signal Process, vol. 17, no. 2, pp. 103118, 2003.

[5] S. Kim, S.A. Campbell, and X. Liu, "Stability of a Class of Linear Switching Systems With Time Delay," IEEE Trans. on Circuits and Systems-I: Regular Papers, vol. 53, no. 2, pp. 384-393, 2006.

[6] Z. Sun, and S.S. Ge, "Analysis and synthesis of switched linear control systems," Automatica, vol. 41, no. 2, pp. 181-195, 2005.

[7] K. Wulff, Quadratic and Non-Quadratic Stability Criteria for Switched Linear Systems, Ph.D Thesis, National University of Ireland, 2004.

[8] M.S. Branicky, "Stability of switched and hybrid systems," in: Proc. the $33^{\text {rd }}$ IEEE Conf. on Decision and Control, pp. 3498-3503, 1994.

[9] Z. Sun, S. S. Ge, and T. H. Lee, "Controllability and reachability criteria for switched linear systems," Automatica, vol. 38, no. 5, pp. 775-786, 2002.

[10] J.P. Hespanha, D. Liberzon, D. Angeli, and E.D. Sontag, "Nonlinear norm-observability notions and stability of switched systems," IEEE Trans. Automat. Control, vol. 52, no. 2, pp. 154-168, 2005.

[11] S. Solmaz, R. Shorten, K. Wulff ve F.Ó. Cairbre, "A design methodology for switched discrete time linear systems with applications to automotive roll dynamics control," Automatica, vol. 44, no. 9, pp. 2358-2363, 2008.

[12] X. Xu and P. J. Antsaklis, "Optimal control of switched systems based on parameterization of the switching instants," IEEE Trans. Automat. Control, vol. 49, no. 1, pp. 2-16, 2004.

[13] K. Wulff, F. Wirth, and R. Shorten, "A control design method for a class of switched linear systems," Automatica, vol. 45, no. 11, pp. 2592-2596, 2009

[14] C. Chen, S. Fei, K. Zhang, and Y. Lu, "Control of switched linear systems with actuator saturation and its applications," Mathematical and Computer Modelling, vol. 56, no. 1-2, pp. 14-26, 2009.
[15] S.H. HosseinNia, I. Tejado, B.M. Vinagre, "Robust Fractional order PI Controller for Switching Systems," in: Proc. the $5^{\text {th }}$ Symp. on. Fractional Differentiation and its Applications (FDA'2012), Hohai University, 2012.

[16] Y.I. Neimark, "D-decomposition of the space of quasi-polynomials (on the stability of linearized distributive systems)," American Mathematical Society Translations, vol. 102, pp. 95-131, 1973.

[17] D. Liberzon, ve A.S. Morse, "Basic problems in stability and design of switched systems," IEEE Control Systems Magazine, vol. 19, pp. 59-70, 1999 .

[18] S.H. HosseinNia, I. Tejado, B.M. Vinagre, "A method for the design of robust controllers ensuring the quadratic stability for switching systems," J. of Vibration and Control, vol. 20, no. 7, pp. 1085-1098, 2014.

[19] I. Podlubny, "Fractional Order Systems and $\mathrm{PI}^{\lambda} \mathrm{D}^{\mu}$ Controllers," IEEE Trans. on Automatic Control, vol. 44, no. 1, pp. 208-214, 1999.

[20] J. Hwang, J.-F. Leu, and S.-Y. Tsay, "A note on time-domain simulation of feedback fractional-order systems," IEEE Trans. on Automatic Control, vol. 47, no. 4, pp. 625-631, 2002.

[21] J. Ackermann, D. Kaesbauer, "Design of robust PID controllers," in: Proc. the European Control Conference, pp. 522-527, 2001.

[22] M.-T. Ho, A. Datta, and S.P. Bhattacharyya, "A new approach to feedback stabilization," in: Proc. The $35^{\text {th }}$ Conf. on Decision and Control, Kobe, Japan, 1996.

[23] İ. Işık, and S.E. Hamamci, "Anahtarlamalı Sistemleri Kararlı Yapan PI Kontrolör Setinin Hesab1," in: Proc. the TOK 2013 Turkish Automatic Control National Meeting, Malatya, Turkey, 2013, (in Turkish).

[24] Y.C. Cheng, and C. Hwang, "Stabilization of unstable first-order timedelay systems using fractional-order PD controllers," $J$. of the Chinese Inst. of Engineers, vol. 29, pp. 241-249, 2006.

\section{BIOGRAPHIES}

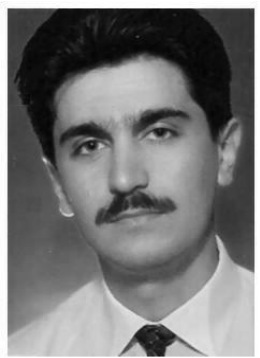

SERDAR E. HAMAMCI received the B.S. degree in electronics engineering from Erciyes University in 1992 and the M.S. and Ph.D. degrees in electrical-electronics enginnering from Firat University in 1997 and 2002 respectively.

From 1993 to 2009, he was a Research Assistant with the Electrical-Electronics Engineering Department at Inonu University. Since 2009, he has been an Associate Professor with the same department. His research interest includes control system design, stabilization and fractional order systems. 